\title{
249 CHARACTERIZATION OF UNRESECTABLE AND/OR METASTATIC SOLID TUMORS PATIENTS TREATED WITH SYSTEMIC ANTICANCER THERAPY IN FIRST-LINE SETTINGS: A PAN-TUMOR COMMUNITY-ONCOLOGY BASED STUDY
}

${ }^{1}$ Elise Wu, ${ }^{2}$ Sneha Sura*, ${ }^{3}$ Alexander Spira. ${ }^{1}$ Merck and Co., Inc., Kenilworth, NJ, USA; ${ }^{2}$ McKesson Life Sciences, The Woodlands, TX, USA; ${ }^{3}$ Texas Oncology, Fairfax, VA, USA

Background Since the introduction of immunotherapy (I/O) in 2014, increasing number of patients with solid tumor are treated with I/O. This study aimed to describe demographic and clinical characteristics of patients initiating $\mathrm{I} / \mathrm{O}$ versus non-I/O-based systemic anticancer therapy (SACT) in the firstline (1L) settings among patients diagnosed with unresectable and/or metastatic solid tumors in a real-world US community oncology setting from pan-tumor perspective.

Methods This retrospective observational study used the US Oncology Network's iKnowMed electronic health record database and included adults diagnosed with unresectable and/or metastatic solid tumors who initiated SACT. We selected 11 solid tumors in the cohort because $\mathrm{I} / \mathrm{O}$ has been approved as $1 \mathrm{~L}$ treatment for unresectable and/or metastatic tumors prior to August 2019. We included I/O indicated patients who initiated either $1 \mathrm{~L} \mathrm{I/O}$ or non-I/O-based SACT between 01/01/ 2014 and 08/31/2019. All analysis was performed based on pan-tumor perspective with no stratification by tumor types. Descriptive statistic was conducted to characterize demographic and clinical characteristics among those who used $\mathrm{I} / \mathrm{O}$ versus non-I/O-based SACT.

Results A total of 20,057 patients were included in the study cohort. 5,119 (25.5\%) used I/O and 14,938 (74.5\%) used non-I/O-based SACT. The use of $\mathrm{I} / \mathrm{O}$ increased from $4.0 \%$ in 2014 to $66.3 \%$ in 2019 with a corresponding decline in non$\mathrm{I} / \mathrm{O}$ use during the same period. Patients who initiated $\mathrm{I} / \mathrm{O}$ were older (69.4 vs. 67.8 years, $\mathrm{p}<0.0001)$, and a greater proportion were male $(57.8 \%$ vs. $53.0 \%, \mathrm{p}<0.0001)$ and had Medicare insurance $(57.0 \%$ vs. $55.2 \%, \mathrm{p}=0.025)$ compared to those who initiated non-I/O-based SACT. Race distribution and smoking status were significantly different between $\mathrm{I} / \mathrm{O}$ users and non-users $(\mathrm{p}<0.0001$ for both). The mean duration of time from unresectable and/or metastatic solid tumors diagnosis to SACT initiation was higher in $\mathrm{I} / \mathrm{O}$ users compared to non-I/O-based SACT users (10.1 weeks vs. 6.1 weeks, $\mathrm{p}<0.0001)$. Among clinical characteristics, a greater proportion of $\mathrm{I} / \mathrm{O}$ users had ECOG performance status of $3+(2.1 \%$ vs. $1.6 \%, \mathrm{p}<0.0001)$, lung as a site of metastases $(13.9 \%$ vs. $10.1 \%, \mathrm{p}<0.0001)$, and had $1+$ Charlson comorbidities (19.2\% vs. $15.6 \%, p=0.002)$.

Conclusions This real-world study provides insight into characteristics of patients with unresectable and/or metastatic solid tumors who initiated SACT in real-world oncology settings. Patients who received I/O appeared to be older, and sicker and takes a longer time to receive treatment since diagnosis compared to those who received non-I/O-based SACT. Future research should carefully adjust patients' characteristics while evaluating the effect of $\mathrm{I} / \mathrm{O}$ therapy on clinical and economic outcomes.

Trial Registration The authors would like to acknowledge Lisa Kaspin-Powell, PhD, ELS, an employee of Ontada, for editorial assistance, which was funded by Merck \& Co., Inc.

http://dx.doi.org/10.1136/jitc-2021-SITC2021.249 